\title{
Is there a response shift in generic health-related quality of life 6 months after glioma surgery?
}

\author{
Asgeir Store Jakola ${ }^{1,2,3} \cdot$ Ole Solheim $^{3,4,5} \cdot$ Sasha Gulati ${ }^{3,5,6} \cdot$ Lisa Millgård Sagberg ${ }^{3,4,5}$
}

Received: 29 September 2016 / Accepted: 23 November 2016/Published online: 7 December 2016

(C) The Author(s) 2016. This article is published with open access at Springerlink.com

\begin{abstract}
Background Patients may recalibrate internal standards when faced with a serious diagnosis or neurological deficits. This so-called response shift is important to understand in longitudinal health-related quality of life (HRQoL) data, but this is not quantitatively assessed in glioma patients.

Methods Patients with gliomas were eligible for this HRQoL study. We used EuroQol-5D $3 \mathrm{~L}$ to assess generic HRQoL with assessment preoperatively and at 6 months postoperatively. At time of follow-up, patients scored how they considered their baseline HRQoL in retrospect using the same questionnaire ("then-test").

Results Seventy-three patients were enrolled between January 2013 and September 2015. With the then-test approach, the mean EQ-5D $3 \mathrm{~L}$ index was similar compared to baseline (0.77, mean difference $0.01,95 \%$ CI -0.57 to $0.07, p=$ 0.82). Also, then-test and baseline VAS score were similar (mean difference $0,95 \% \mathrm{CI}-7$ to $7, p=0.97$ ). However, a $0.10-0.13$ difference from baseline was observed in patients that improved or deteriorated in HRQoL at follow-up according to the then-test EQ-5D $3 \mathrm{~L}$ index value. The direction of
\end{abstract}

Asgeir Store Jakola

legepost@gmail.com

Ole Solheim

ole.solheim@ntnu.no

Sasha Gulati

sashagulati@hotmail.com

Lisa Millgård Sagberg

lisa.millgard.sagberg@ntnu.no

1 Department of Neurosurgery, Sahlgrenska University Hospital, Blå Stråket 5, vån 3, 41345 Göteborg, Sweden change as observed from the then-test was similar to the direction of clinical change, reducing the impact of any HRQoL change from baseline to follow-up.

Conclusions On average, we observed no response shift using EQ-5D $3 \mathrm{~L}$ in the selection of glioma patients able to participate at 6 months after surgery. However, following change in HRQoL at follow-up, response shift seems to reduce the effects of HRQoL changes by lowering of internal standards in patients that deteriorate and raising the standards in patients that improve.

Keywords Glioma $\cdot$ Neurosurgery $\cdot$ Quality of life $\cdot$ Brain neoplasm $\cdot$ Surgical management

\section{Introduction}

Research on health-related quality of life (HRQoL) in glioma patients is gaining interest $[14,18,22]$. However, so-called response shift is frequently considered to interfere with interpretation of longitudinal HRQoL data [11, 14, 18]. Response shift refers to the phenomenon where patients score better
2 Institute of Neuroscience and Physiology, Sahlgrenska Academy, University of Gothenburg, Gothenburg, Sweden

3 Department of Neurosurgery, St. Olavs Hospital, Trondheim, Norway

4 Norwegian Advisory Unit for Ultrasound and Image-Guided Therapy, St. Olavs University Hospital, Trondheim, Norway

5 Department of Neuroscience, Norwegian University of Science and Technology, Trondheim, Norway

6 Norwegian Centre of Competence in Deep Brain Stimulation for Movement Disorders, St. Olavs University Hospital, Trondheim, Norway 
because over time they adapt to a new situation; for instance, a handicap or an illness $[17,18]$. It is considered to involve elements of recalibration of internal standards, change in priorities and/or a different view upon the concept of HRQoL [3, $8,27]$. In patients with gliomas all these elements may change when faced with neurological deficits or being diagnosed with a life-threatening illness [18].

Thus, such recalibration may therefore affect longitudinal HRQoL data and interpretation of results, but unless assessed directly it is impossible to detect if a recalibration has occurred [3]. The most common approach to study response shift is the socalled "then-test approach" where respondents retrospectively score how they at time of follow-up consider their earlier or baseline HRQoL in the light of their new situation [17, 25, 27].

In a meta-analysis from 2006, the effect sizes of response shift were found to be small, with the largest effect sizes detected for fatigue and global HRQoL [25]. Even though response shift is known to occur in cancer patients [8, 9, 17, 18], we have not found response-shift studies in glioma patients. Searching for clinically relevant subgroups that could exhibit response shift is also indicated.

In this project we aimed to prospectively study the direction and magnitude of response shift in glioma patients using a global, generic HRQoL measure. Further, since response shift is most pronounced in the presence of a trigger [2] (i.e. less likely to occur in patients with stable condition), we explore response shift in patients with a significant HRQoL change at follow-up.

\section{Materials and methods}

\section{Study population}

All adult patients ( $\geq 18$ years old) that underwent surgery for glioma at St. Olavs University Hospital, Trondheim, Norway, in the period from January 2013 through September 2015 were eligible for inclusion in this study. In this period 210 patients with glioma underwent a neurosurgical procedure of biopsy or resection. As shown in the flow-chart (Fig. 1), we included in total 73 patients that had both a self-reported baseline assessment and a self-reported postoperative assessment at 6 months with the renewed retrospective scoring of their baseline HRQoL.

All included patients had histopathological diagnosis of WHO grade II-IV glioma confirmed by a neuropathologist according to the 2007 WHO classification [19].

\section{Euroqol 5D 3L}

The EuroQol 5D 3L (EQ-5D 3L) is a generic measure of health-related QoL developed by the EuroQol Group [28]. The EQ-5D 3L has been validated in a Norwegian normal population [21]. The questionnaire has been applied to a wide range of health conditions and treatments.

In EQ-5D 3L, five dimensions of HRQoL are scored: mobility, self-care, usual activities, pain/discomfort and anxiety/ depression, with three possible answers to each dimension, i.e. 'no problem', slight problem' or 'major problem'. This results in the 243 different possible health states, which are transformed into an index value based on a large survey in the UK population [6]. EQ-5D 3L index value is from -0.594 to 1 , where 1 corresponds to perfect health, and 0 to death. Negative values are considered to be worse than death. The questionnaire also contains a visual analogue scale (VAS), reflecting the global health state from 0 (worst possible score) to 100 (best possible score).

We chose to use EQ-5D 3L due to the simplicity of the instrument, to enhance patient perception and perhaps also compliance. We have previously demonstrated that EQ-5D $3 \mathrm{~L}$ index value shows good correlation to Karnofsky Performance Scale (KPS) in patients with gliomas and is responsive to new neurological deficits which is highly relevant in this patient group [15]. Further, we have found the minimal clinically important change (MIC) to be approximately 0.13 0.15 [22]. In this study we decided a priori that 0.15 was the minimum magnitude of change necessary to be a clinical important change.

\section{Data collection}

Our routine has been that patients provided written informed consent and filled out the EQ-5D 3L questionnaire 1-3 days before surgery. The operating surgeon scored preoperative KPS prospectively on admission. Patient follow-up by a study nurse was scheduled at 6 months postoperatively to allow recovery from transient surgically induced deficits. At 6 months, patients undergoing radiotherapy had also had time for recovery; however, tumour progression/recurrence may, on the other hand, occur.

Data were collected from the hospital's electronic patient charts. Patient characteristics and preoperative status including Charlson comorbidity index [5], KPS [16] and eloquence in tumour location [24] were registered. Complications were scored according to the classification system proposed by Landriel Ibañez and co-workers [10]. Tumour volumes and resection grades were determined from preoperative and early postoperative magnetic resonance imaging (MRI) volumes using an ellipsoid model $\left(4 л \times \mathrm{r}^{3} / 3\right)$ where gross total resection (GTR) was defined as $<0.175 \mathrm{~cm}^{3}$ residual tumour tissue on the early $(<72 \mathrm{~h}$ ) postoperative $1.5-\mathrm{T}$ or $3.0-\mathrm{T}$ MRI scans; both techniques previously described by others [26]. If the tumour had a smaller contrast-enhanced region being surrounded by a larger region with T2/FLAIR abnormalities believed to be glioma and not oedema the entire tumour was measured, and not only the contrast-enhancing part. For non-contrast- 
Fig. 1 Flow chart
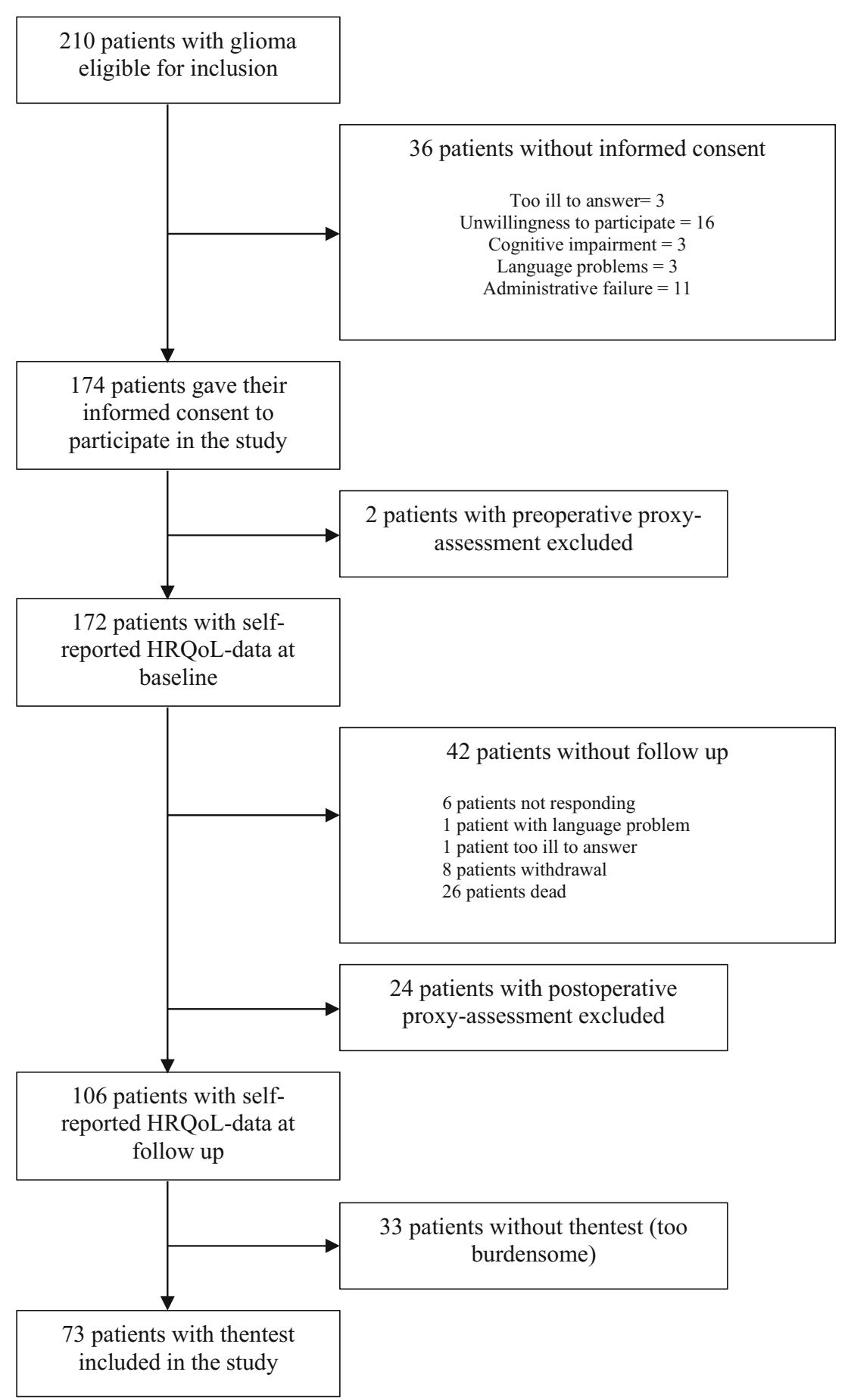

enhancing gliomas, the T2/FLAIR images were used for volume assessment.

\section{Response shift}

To assess response shift, we used a pre-test/post-test design with a then-test [29]. The patients were asked to score their baseline ratings of the five EQ-5D 3L domains and the VAS score immediately after the post-test assessment at 6 months follow-up. The research nurse emphasised that the intention of the retrospective test was not to remember and copy their answers at baseline but to provide a renewed baseline HRQoL as they now would consider it in retrospect (thentest). According to this method, patients use their new internal standards in the then-test. The mean difference between the then-test and prospective baseline test was then calculated to provide the recalibration response shift effect, while the mean difference between the then-test and the post-test was 
calculated to provide a better estimate of the adjusted time effect (i.e. the true change).

\section{Statistical analysis}

To analyse data and to create graphs we used the software package SPSS (version 21.0; SPSS, Chicago, IL, USA). QQ plots were used to test if data were normally distributed. When analysing changes in EQ-5D 3L (e.g. before and after surgery) a paired sample $t$-test was used. Comparisons of continuous data were done with independent samples $t$-test. Categorical data were analysed with Pearson's chi-squared test. A $p$ value $\leq 0.05$ was considered statistically significant.

\section{Results}

Baseline and surgical characteristics are summarised in Table 1. In 43 patients (59\%) the surgery was a primary operation. The histopathology revealed a diffuse low-grade glioma in 26 patients (36\%) and a high-grade glioma in 47 patients (64\%).

Follow-up data at 6 months from baseline are presented in Table 2. Mean EQ-5D $3 \mathrm{~L}$ index value and VAS score at follow-up did not differ from the baseline scores $(p=0.52$ and $p=0.94$, respectively). According to the suggested glioma MIC value for the EQ-5D 3L index [22], we observed that $14(19 \%)$ improved, 43 (59\%) remained unchanged and 16 (22\%) deteriorated from the true baseline test.

Using the then-test approach on the entire sample, we found that the mean EQ-5D 3L index was similar at thentest compared to baseline $(0.77$, mean difference $0.01,95 \%$ CI -0.57 to $0.07, p=0.82$, Fig. 1). Similarly, then-test and baseline VAS score was compared demonstrating a mean difference of 0 (95\% CI -7 to $7, p=0.97)$. Since 30 patients had been operated on previously, we assessed if there was any difference in response shift using EQ-5D 3L index between groups, but there was none (mean difference $0.06,95 \%$ CI -0.08 to $0.21, p=0.40)$. Using the then-test to evaluate change in relation to MIC, we observed that $21 \%$ improved, $54 \%$ remained unchanged and $26 \%$ deteriorated. Thus, in this sample of glioma patients, we observed on average no indication of any statistically significant or clinically relevant response shift.

\section{Patients with minimal clinical important change}

We found that the 16 patients who reported clinically significant deterioration in EQ-5D 3L index at 6 months compared with true baseline values reported a difference of 0.13 in the then-test versus true baseline, a result that indicate that their baseline HRQoL was considered to be worse when scored in
Table 1 Baseline characteristics

\begin{tabular}{ll}
\hline & $n=73$ \\
\hline Age in years, mean (SD) & $49(15)$ \\
Female, $n(\%)$ & $26(36)$ \\
Preoperative KPS $\geq 70, n(\%)$ & $70(96)$ \\
Preoperative CCI $>1, n(\%)$ & $3(4)$ \\
Symptoms, $n(\%)$ & \\
Headache & $24(33)$ \\
Seizures & $27(37)$ \\
Cognitive & $17(26)$ \\
Dysphasia & $6(8)$ \\
Motor & $3(4)$ \\
Primary surgery, $n(\%)$ & $43(59)$ \\
Preoperative use of corticosteroids, $n(\%)$ & $28(38)$ \\
Preoperative tumour volume, median (IQR) & $24.2(5.7-43.9)$ \\
Eloquent, $n(\%)$ & $21(29)$ \\
Resection, $n(\%)$ & $70(96)$ \\
Histopathology, $n(\%)$ & \\
WHO grade II & $26(36)$ \\
WHO grade III & $19(26)$ \\
WHO grade IV & $28(38)$ \\
Extent of resection, median (IQR) & $93.2(81.7-100)$ \\
Baseline EQ-5D 3 L index value, mean (SD) & $0.78(0.24)$ \\
Baseline EQ-VAS, mean (SD), $n=67$ & $73(21)$ \\
\hline
\end{tabular}

KPS Karnofsky Performance Scale score, CCI Charlson comorbidity index, Eloquent refers to Sawaya grade 3

retrospect. However, in these patients the difference between the true VAS at baseline and the then-test VAS was 0 .

When analysing those who improved $(n=14)$ at 6 months compared to true baseline, they had a 0.10 difference in thentest compared to true baseline, with the direction that indicated that they considered their baseline HRQoL to be better in retrospect.

Similarly, the VAS score was considered 6 points better in then-test than at baseline. In Table 3 we also explored the different EQ-5D 3L domains at then-test compared with baseline assessment. The findings of EQ-5D 3L index value in the different MIC groups are visualised in Figs. 2 and 3 to enhance interpretation.

\section{Exploratory subgroup analyses of patients with clinically significant response shift}

We used the suggested MIC as guidance in defining patients who significantly considered their baseline to be better in 
Table 2 Important variables 6 months postoperatively

\begin{tabular}{lll}
\hline & $n=73$ & Significant change from baseline \\
\hline 6 month KPS $\geq 70, n(\%)$ & $65(89)$ & $p=0.21$ \\
EQ-5D 3 L index value at 6 months, mean (SD) & $0.79(0.21)$ & $p=0.52$ \\
MIC group, $n(\%)$ & NA \\
$\quad$ Better & $14(19)$ & \\
Similar & $43(59)$ & \\
$\quad$ Worse & $16(22)$ & \\
VAS score at 6 months, mean (SD), $n=68$ & $72(17)$ & $p=0.94$ \\
\hline
\end{tabular}

MIC minimal clinically important change; the MIC is set to 0.15 , as reported by Sagberg and co-workers retrospect $(n=18)$ and those who significantly considered their baseline to be worse in retrospect $(n=15)$.

We searched predictors among the variables in Table 1 in addition to significant surgical complications (Landriel Ibañez grade 2 or more) or surgically induced neurological deficits.

Of patients who scored their baseline better in retrospect compared to the rest of the cohort, only seizures at baseline (61\% versus $29 \%, p=0.015)$ and baseline EQ-5D 3L index

Table 3 How the then-test of EQ-5D domains compare with baseline assessment

\begin{tabular}{|c|c|c|c|}
\hline & Better & Similar & Worse \\
\hline \multicolumn{4}{|l|}{ ENTIRE SAMPLE $(n=73)$} \\
\hline Then-test mobility $(n=72)$ & 3 & 67 & 2 \\
\hline Then-test self-care $(n=72)$ & 2 & 69 & 1 \\
\hline Then-test activity $(n=73)$ & 12 & 50 & 11 \\
\hline Then-test pain $(n=70)$ & 14 & 46 & 10 \\
\hline Then-test anxiety $(n=72)$ & 12 & 49 & 11 \\
\hline \multicolumn{4}{|l|}{ MIC IMPROVED $(n=14)$} \\
\hline Then-test mobility $(n=14)$ & 1 & 12 & 1 \\
\hline Then-test self-care $(n=14)$ & 2 & 11 & 1 \\
\hline Then-test activity $(n=14)$ & 2 & 10 & 2 \\
\hline Then-test pain $(n=14)$ & 2 & 10 & 2 \\
\hline Then-test anxiety $(n=14)$ & 7 & 5 & 2 \\
\hline \multicolumn{4}{|l|}{ UNCHANGED $(n=43)$} \\
\hline Then-test mobility $(n=43)$ & 1 & 42 & 0 \\
\hline Then-test self-care $(n=43)$ & 0 & 43 & 0 \\
\hline Then-test activity $(n=43)$ & 10 & 26 & 7 \\
\hline Then-test pain $(n=41)$ & 11 & 27 & 3 \\
\hline Then-test anxiety $(n=42)$ & 3 & 33 & 6 \\
\hline \multicolumn{4}{|l|}{ MIC DETERIORATED $(n=16)$} \\
\hline Then-test mobility $(n=15)$ & 1 & 13 & 1 \\
\hline Then-test self-care $(n=15)$ & 0 & 15 & 0 \\
\hline Then-test activity $(n=16)$ & 0 & 14 & 2 \\
\hline Then-test pain $(n=15)$ & 1 & 9 & 5 \\
\hline Then-test anxiety $(n=16)$ & 2 & 11 & 3 \\
\hline
\end{tabular}

Better in this context refer to that the then-test indicate fewer symptoms/ problems than the baseline assessment value $(0.63$ versus $0.82, p=0.003)$ were factors associated with significant response shifts.

Of patients who scored their baseline worse in retrospect compared to the rest of the cohort, only significant surgical complications was an associated factor (27 versus $3 \%, p=$ 0.004).

\section{Discussion}

Response-shift assessment of baseline EQ-5D 3L index value and VAS score at 6 months following surgery for glioma patients using the then-test approach revealed that in our selection of patients there was on average no response shift. This may be due to the fact that the vast majority of our respondents were clinically stable at follow-up as assessed with EQ-5D 3L. Another factor contributing to averaging of results was that we had almost similar proportion of patients improving (19\%) and deteriorating (22\%) in HRQoL at 6 months from baseline. Response shifts were more frequently observed in both patients that improved and patients that deteriorated according to the MIC, but the direction was opposite. Patients that improved had raised their internal standards, while patients that deteriorated had lowered their internal standards.

Further, we explored possible factors associated with significant response shift. A low baseline HRQoL is likely to be considered better in retrospect according to these exploratory analyses. This may possibly relate to ceiling effects of generic HRQoL measures at baseline, where improvement of maximum score is impossible, or simply due to regression of the mean [22]. Also, patients with seizures at baseline were overrepresented in the group that reported better baseline HRQoL in retrospect. These patients are likely to have improved HRQoL at follow-up due to chance of seizure freedom with extensive surgery, and therefore now perhaps forgot or repressed how it was living with seizures [4].

As observed by others studying other conditions, response shift is only present in case of a catalyst [2], and in this regard it is apparently not enough to be diagnosed with a glioma or facing repeated surgery due to recurrence, as we only observed response shift in relation to significant changes in 
Fig. 2 Response shift assessed in the entire sample $(n=73)$

Fig. 3 Response shift seen in EQ-5D 3L index value in patients according to groups based on minimal clinically important change
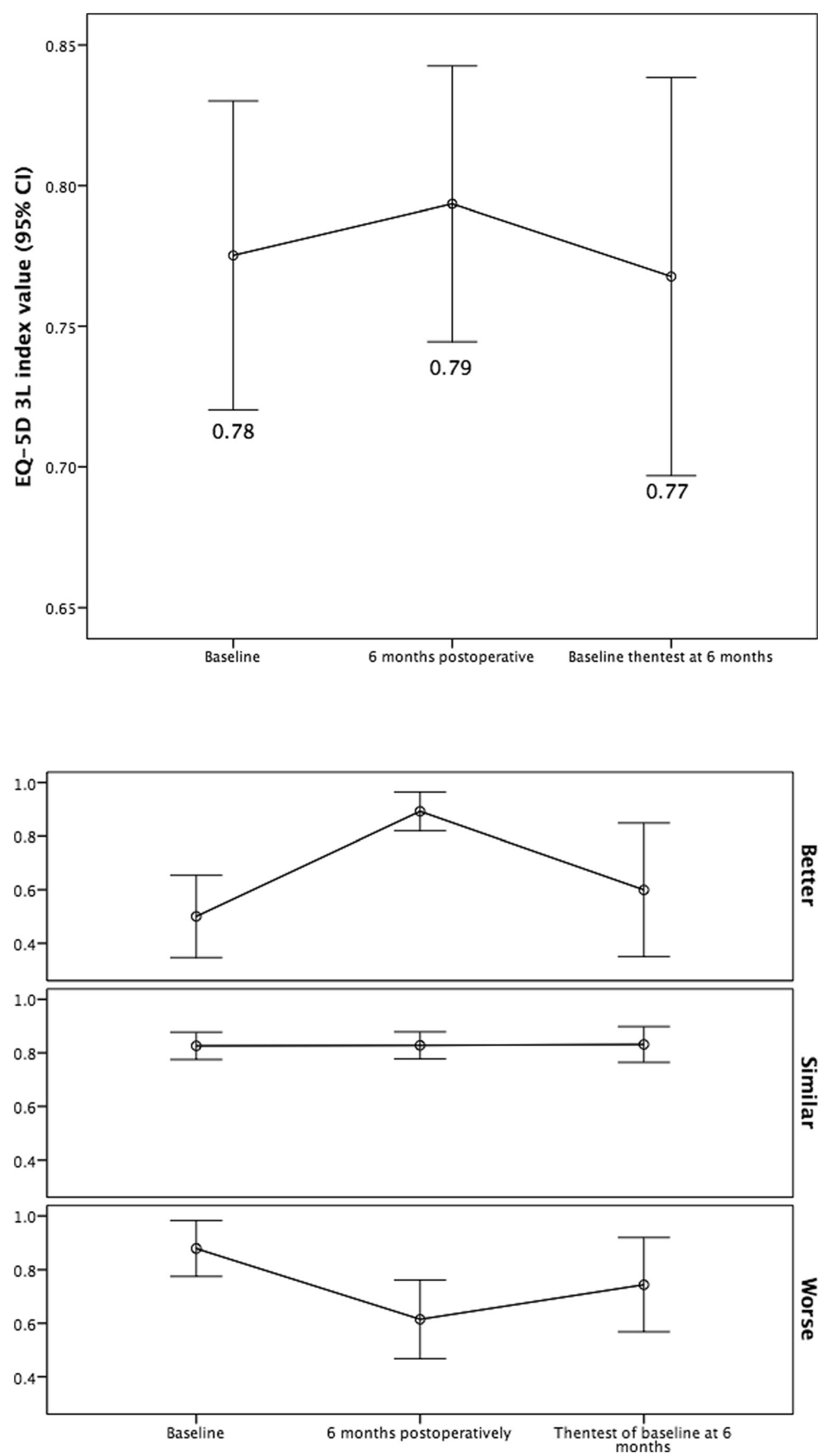
HRQoL after surgery. However, we observe that significant complications triggered a response shift in terms of considering the baseline HRQoL worse in retrospect, reducing the negative impact on HRQoL from the surgical complications 6 months after surgery.

As seen above, the direction of response shift in our study suggests that response shift may reduce the actual changes seen in longitudinal HRQoL studies, with respect to both deterioration and improvement, when using EQ-5D 3L index value according to the then-test model [25]. One practical implication this has for interpretation is that glioma patients who remain stable after surgery according to MIC groups are not stable simply due to response shift since we did not observe any response shift in this stable group. Further, for glioma patients with significant changes in HRQoL after surgery, they seemingly more frequently recalibrate their internal standards, reducing the actual effect size of the change as observed in longitudinal studies.

Our study was not designed to evaluate the relative importance of the different elements of response shift (i.e. recalibration, reprioritisation and reconceptualisation). In a recent study of patients with prostate cancer, reconceptualisation was not an important factor, perhaps indicating that the other two are more important elements in response shift among cancer patients [8]. Others consider the elements of reprioritisation and reconceptualisation not to be a true response shift, but rather coping strategies affecting the true value [3]. In this view, recalibration is the only true response shift.

The underlying assumptions of the then-test used in this study have recently been criticised [27]. Most importantly, the assumption of cognitive consistency of respondents at the different time points may not hold true [27]. Also, recall bias of previous health condition is a concern $[25,27]$, especially since this may be more pronounced in patients with cognitive deficits [1]. In our study, all patients were able to complete the thentest, but many were naturally cognitively impaired. This may contribute to the rather wide confidence intervals observed in this study [3]. Even though global HRQoL has been found to have among the larger effect sizes, generic HRQoL measures are in general less sensitive and this may have contributed to the group level results in our study [25]. Also, since response shift assessments require patients that are able to report their own health state at both baseline and at follow-up, thus terminally ill patients, patients with severe cognitive deficits or patients with severe language problems were not included in this study. Consequently, the interpretation of our results must be understood in the light of the selection reported in Fig. 1, where an unavoidable selection bias seems to be present which may influence our results.
Finally, in the light of the above-mentioned limitations and since this is the first paper to assess response shift after glioma surgery caution is needed when interpreting our results, and especially the more exploratory findings should be considered hypothesis-generating.

This study is important for the interpretation of our earlier studies using EQ-5D 3L in gliomas [7, 12-15, $22,23]$. Most importantly, we are now more confident that it is not a response-shift artefact when patients reported stable HRQoL. It is perhaps comforting that the response shift may reduce the negative effect of glioma surgery or disease progression, but we should not accept that patients reduce their standards if this can be avoided with either safer surgery or more effective treatment delaying time to progression. Thus, we should continue to evaluate our results and readjusting our practice if this improves the onco-functional balance [20].

\section{Conclusions}

Our results indicate that response shift in glioma patients undergoing surgery is dependent on changes in HRQoL at time of assessment. Importantly, patients reporting stable HRQoL at follow-up demonstrated no response shift in our study. Further, response shift seems to reduce the effects of HRQoL changes by lowering of internal standards in patients that deteriorate and raising the standards in patients that improve.

Acknowledgements We would like to thank Linda Nordtvedt and Camilla Brattbakk for assisting in data collection.

\section{Compliance with ethical standards}

Ethical approval The Regional Committee for Medical and Health Research Ethics in Central Norway approved the study.

Informed consent Informed consent was obtained from all individual participants included in the study.

Conflict of interest All authors certify that they have no affiliations with or involvement in any organisation or entity with any financial interest (such as honoraria; educational grants; participation in speakers' bureaus; membership, employment, consultancies, stock ownership, or other equity interest; and expert testimony or patent-licensing arrangements), or non-financial interest (such as personal or professional relationships, affiliations, knowledge or beliefs) in the subject matter or materials discussed in this manuscript.

Funding The Norwegian Cancer Society provided financial support in terms of funding to Asgeir S. Jakola (project 5703787). The sponsor had no role in the design or conduct of this research. 
Open Access This article is distributed under the terms of the Creative Commons Attribution 4.0 International License (http:// creativecommons.org/licenses/by/4.0/), which permits unrestricted use, distribution, and reproduction in any medium, provided you give appropriate credit to the original author(s) and the source, provide a link to the Creative Commons license, and indicate if changes were made.

\section{References}

1. Ahmed S, Mayo NE, Wood-Dauphinee S, Hanley JA, Cohen SR (2004) Response shift influenced estimates of change in healthrelated quality of life poststroke. J Clin Epidemiol 57:561-570

2. Ahmed S, Sawatzky R, Levesque JF, Ehrmann-Feldman D, Schwartz CE (2014) Minimal evidence of response shift in the absence of a catalyst. Qual Life Res 23:2421-2430

3. Blome C, Augustin M (2015) Measuring change in quality of life: bias in prospective and retrospective evaluation. Value Health 18:110-115

4. Chang EF, Potts MB, Keles GE, Lamborn KR, Chang SM, Barbaro NM, Berger MS (2008) Seizure characteristics and control following resection in 332 patients with low-grade gliomas. J Neurosurg 108:227-235

5. Charlson ME, Pompei P, Ales KL, MacKenzie CR (1987) A new method of classifying prognostic comorbidity in longitudinal studies: development and validation. J Chronic Dis 40:373-383

6. Dolan P (1997) Modeling valuations for EuroQol health states. Med Care 35:1095-1108

7. Drewes C, Sagberg LM, Jakola AS, Solheim O (2016) Quality of life in patients with intracranial tumors: does tumor laterality matter? J Neurosurg. doi:10.3171/2015.12.JNS152252

8. Gerlich C, Schuler M, Jelitte M, Neuderth S, Flentje M, Graefen M, Kruger A, Mehnert A, Faller H (2016) Prostate cancer patients' quality of life assessments across the primary treatment trajectory: 'True' change or response shift? Acta Oncol 55:814-820

9. Hamidou Z, Dabakuyo-Yonli TS, Guillemin F, Conroy T, Velten M, Jolly D, Causeret S, Graesslin O, Gauthier M, Mercier M, Bonnetain F (2014) Impact of response shift on time to deterioration in quality of life scores in breast cancer patients. PLoS One 9:e96848

10. Ibañez FAL, Hem S, Ajler P, Vecchi E, Ciraolo C, Baccanelli M, Tramontano R, Knezevich F, Carrizo A (2011) A new classification of complications in neurosurgery. World Neurosurg 75:709-715

11. Jakola AS, Gulati M, Gulati S, Solheim O (2012) The influence of surgery on quality of life in patients with intracranial meningiomas: a prospective study. J Neurooncol 110:137-144

12. Jakola AS, Gulati S, Weber C, Unsgård G, Solheim O (2011) Postoperative deterioration in health related quality of life as predictor for survival in patients with glioblastoma: a prospective study. PLoS ONE 6:e28592

13. Jakola AS, Sagberg LM, Gulati S, Solheim O (2015) Perioperative quality of life in functionally dependent glioblastoma patients: a prospective study. Br J Neurosurg 29:843-849
14. Jakola AS, Unsgard G, Myrmel KS, Kloster R, Torp SH, Sagberg LM, Lindal S, Solheim O (2014) Surgical strategies in low-grade gliomas and implications for long-term quality of life. J Clin Neurosci 21:1304-1309

15. Jakola AS, Unsgard G, Solheim O (2011) Quality of life in patients with intracranial gliomas: the impact of modern image-guided surgery. J Neurosurg 114:1622-1630

16. Karnofsky DA, Burchenal JH (1949) The clinical evaluation of chemotherapeutic agents in cancer. Columbia University Press, New York, pp 191-205

17. Kvam AK, Wisloff F, Fayers PM (2010) Minimal important differences and response shift in health-related quality of life; a longitudinal study in patients with multiple myeloma. Health Qual Life Outcomes 8:79

18. Liu R, Page M, Solheim K, Fox S, Chang SM (2009) Quality of life in adults with brain tumors: current knowledge and future directions. Neuro Oncol 11:330-339

19. Louis DN, Ohgaki H, Wiestler OD, Cavenee WK, Burger PC, Jouvet A et al (2007) The 2007 WHO classification of tumours of the central nervous system. Acta Neuropathol 114:97-109

20. Mandonnet E, Duffau H, Bauchet L (2012) A new tool for grade II glioma studies: plotting cumulative time with quality of life versus time to malignant transformation. J Neurooncol 106:213-215

21. Nord E (1991) EuroQol: health-related quality of life measurement. Valuations of health states by the general public in Norway. Health Policy 18:25-36

22. Sagberg LM, Jakola AS, Solheim O (2014) Quality of life assessed with EQ-5D in patients undergoing glioma surgery: what is the responsiveness and minimal clinically important difference? Qual Life Res 23:1427-1434

23. Sagberg LM, Solheim O, Jakola AS (2016) Quality of survival the 1 st year with glioblastoma: a longitudinal study of patient-reported quality of life. J Neurosurg 124:989-997

24. Sawaya R, Hammoud M, Schoppa D, Hess KR, Wu SZ, Shi W-M, Wildrick DM (1998) Neurosurgical outcomes in a modern series of 400 craniotomies for treatment of parenchymal tumors. Neurosurgery 42:1044-1055

25. Schwartz C, Bode R, Repucci N, Becker J, Sprangers M, Fayers P (2006) The clinical significance of adaptation to changing health: a meta-analysis of response shift. Qual Life Res 15:1533-1550

26. Stummer W, Pichlmeier U, Meinel T, Wiestler OD, Zanella F, Reulen H-J (2006) Fluorescence-guided surgery with 5aminolevulinic acid for resection of malignant glioma: a randomised controlled multicentre phase III trial. Lancet Oncol 7:392-401

27. Taminiau-Bloem EF, Schwartz CE, van Zuuren FJ, Koeneman MA, Visser MR, Tishelman C, Koning CC, Sprangers MA (2016) Using a retrospective pretest instead of a conventional pretest is replacing biases: a qualitative study of cognitive processes underlying responses to thentest items. Qual Life Res 25:1327-1337

28. EuroQol Group (1990) EuroQol - a new facility for the measurement of health-related quality of life. Health Policy 16:199-208

29. Visser MR, Oort FJ, Sprangers MA (2005) Methods to detect response shift in quality of life data: a convergent validity study. Qual Life Res 14:629-639 